\title{
TEREDINIDAE (MOLLUSCA - BIVALVIA) DO RIO MANGUABA E DA PRAIA DE BARREIRAS DO BOQUEIRÃO, PORTO DE PEDRAS E JAPARATINGA, ALAGOAS, BRASIL.
}

\author{
Liriane Monte FREITAS \\ Rosa de Lima Silva MELLO ${ }^{2}$ \\ ${ }^{1}$ LABMAR-UFAL \\ ${ }^{2}$ Departamento de Pesca UFRPE
}

RESUMO

O trabalho consta como primeiro registro de distribuição de moluscos da família Teredinidae (perfuradores de madeira) no litoral do Estado de Alagoas. Os espécimens foram coletados na Região Norte, em áreas de estuário e de manguezal do rio Manguaba e marinha (mediolitoral) na praia de Barreiras do Boqueirão nos Municípios Japaratinga e Porto de Pedras (09 $09^{\circ}$ 27' - $09^{0} 09^{\prime} 19^{\prime \prime}$ 'S e $35^{0} 20^{\prime} 00^{\prime \prime}$ $35^{\circ} 16^{\prime} 33^{\prime}$ ' W). Os exemplares foram coletados de troncos de espécies nativas (coqueiro e mangue) encontrados nos dois ambientes e também de "iscas" artificiais (coletores laminados de pinho). Foram identificadas oito espécies de duas subfamílias: cinco de Teredininae e três de Bankiinae. Do ambiente estuarino - manguezal foram registradas: Nausitora fusticula (51\%), Neoteredo reynei (19\%), Teredo bartschi (17\%), Bankia fimbriatula (8\%) e Lydorus floridanus (1\%). No ambiente marinho (mediolitoral) Teredo clappi (71,3\%), Noteredo sp $(23,4 \%)$ e Bankia fimbriatula $(5,3 \%)$, esta última, a única a estar presente nos dois ambientes.

Palavras-chave: Teredinidae, Mollusca, Bivalvia, Marinho, Tropical

\section{ABSTRACT \\ Teredinidae (Mollusca - Bivalvia) of the River Manguaba and from Barreiras do Boqueirão Beach, Porto de Pedras and Japaratinga, Alagoas, Brazil}

This work presents the distribution of molluscs of the family Teredinidae (wood borers) from the littoral of Alagoas State, Brazil. The specimens were collected at the Northern region in the estuarine and mangrove areas of Manguaba river and in marine areas (intertidal) of Barreiras do Boqueirão Beach located in Japaratinga and

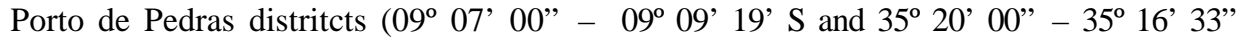
$\mathrm{W})$. The specimens were collected from bark of native trees (coconut and mangrove trees) in both environments and also from artificial attractants (pieces of pine). Eight species of two subfamilies were identified: five Teredininae subfamily and three Bankiinae. In the estuarine-mangrove environment were registere Nausitora fusticula (51\%), Neoteredo reynei (19\%), Teredo bartschi (17\%), Bankia fimbriatula (8\%), and Lyrodus floridanus (1\%). In the marine environment were collected Teredo clappi (71,3\%), Nototeredo sp (23,4\%) and Bankia fimbriatula $(5,3 \%)$. Bankia fimbriatula_. The last one was the unique detected in both environments.

Key words: Teredinidae, Mollusca, Bivalvia, Marine, Tropical 


\section{INTRODUÇÃO}

Os Teredinidae são moluscos bivalves perfuradores de madeira de ambientes aquáticos, sendo parcela importante na manutenção do equilíbrio ecológico através da reciclagem biológica de madeiras nos mares, baías e estuários. Auxiliam no livre fluxo das correntes estuarinas, ao tempo em que atuam nas cadeias alimentares detritívoras entre bactérias e outros organismos, sendo também fonte alimentar para peixes, aves e mamíferos.

A presença de madeira e os parâmetros hidrológicos de salinidade e temperatura, constituem os fatores condicionantes de maior importância para a distribuição das espécies desse grupo, que ocorre principalmente na região entre marés até cerca de 150 metros, embora já tenha sido detectada sua presença à profundidades de 7.488 metros (TURNER,1966). Os Teredinidae são cosmopolitas ocorrendo em todos os mares e oceanos, mas apresentam maior distribuição nas regiões tropicais e subtropicais (TURNER, 1966; NAIR \& SARASWATHY, 1971).

A familia Teredinidae está atualmente organizada em três subfamilias, 15 gêneros e 68 espécies (TURNER, 1971; HOAGLAND e TURNER, 1981), estando registradas para o Brasil 25 espécies distribuidas entre as subfamilias Teredininae e Bankiinae.

O trabalho tem por objetivo contribuir para o conhecimento dos Teredinidae através do inventário das espécies ocorrentes no Estuário - Manguezal do Rio Manguaba e região costeira próxima representada pelo ambiente marinho (mediolitoral) da praia de Barreiras do Boqueirão, ambos localizados nos municípios alagoanos de Porto de Pedras e Japaratinga,. Apresenta pela primeira vez considerações taxonômicas e aspectos ecológicos desse grupo de perfuradores aquáticos para o litoral do Estado de Alagoas.

\section{MATERIAL E MÉTODOS}

As atividades para amostragem de campo foram efetuadas entre outubro de 1991 e março de 1993 no Estuário-Manguezal do Rio Manguaba e na Praia de Barreiras do Boqueirão (Alagoas). Os espécimens foram obtidos a partir de troncos naturais como também de coletores laminados de pinho: as coletas em troncos naturais foram realizadas em cinco estações (Estações I, II, III, IV, VII) ao longo da margem fluvial e em uma estação (Estação VI) em área de praia (mediolitoral); a coleta em coletores foi efetuada por motivos técnicos apenas na estação de desembocadura (Estação V). Todas as coletas foram acompanhadas por verificação dos fatores físico-químicos relacionados à temperatura da água e nível salino de superfície e fundo. Os bivalves foram retirados da madeira com auxílio de faca e machadinha e em seguida fixados em formol a $4 \%$ por 24-72 horas e preservados em álcool a $85 \%$ glicerinado de $1 / 8$. Para avaliação quantiqualitativa foram considerados os exemplares inteiros (valvas - partes moles - paletas) e exemplares com região posterior intacta (sifões-paletas). Para avaliação do comprimento geral das espécies estudadas considerou-se apenas exmplares inteiros (valvas-partes moles-paletas).

A identificação específica foi baseada principalmente em TURNER (1966; 1971) pelo exame das paletas dos moluscos ao microscópio estereoscópio em luz incidente e transmitida 


\section{RESULTADOS}

$\mathrm{Na}$ área estuarina do Rio Manguaba foram identificadas quatro espécies de Teredinidae nas coletas em troncos naturais (Neoteredo reynei Bartsch,1920; Psiloteredo healdi (Bartsch,1931); Nausitora fusticula (Jeffreys, 1860); Bankia fimbriatula Moll e Roch, 1931) e três espécies nas coletas em coletores de pinho (Teredo bartschi Clapp,1923; Lyrodus floridanus (Bartsch, 1922); Bankia. fimbriatula). Em troncos naturais no ambiente marinho (mediolitoral), foram identificadas as espécies, Teredo clappi Bartsch, 1923, Nototeredo sp e Bankia fimbriatula,

\section{Chave para Identificação das Espécies}

1- Paletas com lâminas calcárias, sólidas, sem segmentação, compacta............................. 2

1"- Paletas com lâminas calcárias, segmentadas, não compactas...................................... 6

2- Lâminas pesadas, muito desenvolvidas, em forma de remo largo, com depressão na porção distal da face externa. .3

2"- Lâminas frágeis, pouco desenvolvidas, em forma de remo de configuração variada, sem depressão na porção distal da face externa.

3- Porção distal da face externa da lâmina, com depressão unguiculada e com desgastes, face interna com coloração esbranquiçada; duas dobras carnosas - lapelas - em ambos os lados da região posterior do corpo. Neoteredo reynei

3"- Porção distal da face externa da lâmina com depressão unguiculada, lisa (polida), face interna de coloração laranja claro uniforme; sem lapelas Psiloteredo healdi

4- Lâminas com forma de remo estreito, divisão mediana transversal, formando espécies de "ampulheta" (duas taças em posição invertida) extremidade distal em forma de U típico: perióstraco marrom muito escuro, ausência de larvas pedivéliger ..Lyrodus floridanus

4"- Lâminas em forma de remo largo, ligeiramente ovaladas, com sulco de profundidade e largura variada na extremidade distal; perióstraco amarelo-claro ou ferruginoso 5

5- Sulco transversal em ambas as faces da lâmina, porção distal com coloração amareloclaro Teredo bartschi

5'- Sulco (pequena abertura) na face externa com divisão circular em forma de anel e de coloração ferruginosa na porção distal Teredo clappi

6- Lâminas segmentadas com segmentos fusionados pouco visíveis. 7

6'- Lâminas segmentadas, segmentos distintos, unidos, porém destacáveis; pedúnculo longo. Bankia fimbriatula

7- Lâmina de forma oval, com segmentos fusionados, porém formando espécies de "gomos" circulares visíveis (em luz transmitida); prolongamentos em ambos os lados da extremidade distal geralmente bem nítidos; ausência de tentáculos nos sifões Nototeredo $\mathrm{sp}$

7'- Lâmina de forma variável, geralmente alongada e com incrustações sob a forma de papilas brancas (calcárias); pedúnculo longo; sifão inalante com doze tentáculos ramificados característicos Nausitora fusticula 
Subfamília Terdininae Rafinesque, 1815

Neoteredo reynei (Bartsch, 1920)

Figura 1. Neoteredo reynei 1-5, faces externa e interna de cinco pares de paleta. Sinônimos:Teredo (Neoteredo) reynei Bartsch, 1920(BARTSCH, 1920: 69; 1922: 30).

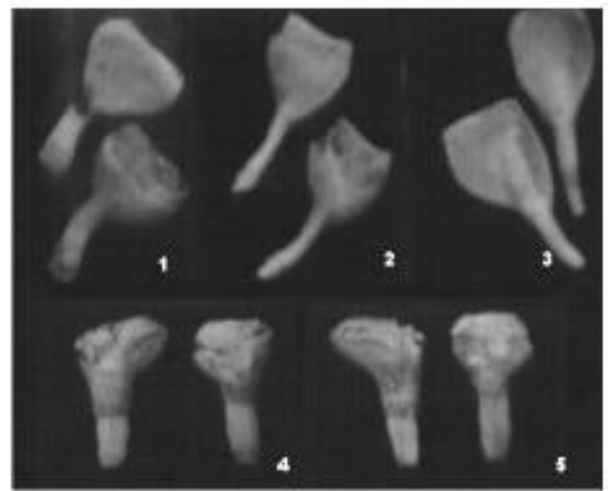

Teredo adami Moll, 1941 (Moll, 1941: 183 apud CLAPP \& KENK, 1963: 698; TURNER, 1966: 86).

Localidade tipo: Paramaribo, Guiana Holandesa (BARTSCH, 1920: 69).

Material examinado: Foram examinados 229 espécimens coletados em cinco estações

Descrição: Partes moles de coloração escura, acinzentada. Paletas ovaladas, calcárias; lâminas compactas de consistência sólida revestida por perióstraco de coloração castanha; face externa com depressão distal unguiculada, com desgastes; pedúnculo curto, calcário, as vezes suavemente sinuoso. Sifões unidos até a porção mediana, de aparência carnosa, apresentando nas extremidades papilas de coloração marrom escuro com numerosos pequenos tentáculos nas bordas da margem interna; sifão exalante com tentáculos reduzidos. Ceco longo, bem visível com grande acúmulo de matéria orgânica. Duas dobras carnosas dorsais denominadas lapelas na região posterior. O maior espécime apresentou comprimento de $480 \mathrm{~mm}$ e o menor $40 \mathrm{~mm}$.

Distribuição Geográfica: Atlântico Ocidental: Suriname, Brasil (Pará, Alagoas, São Paulo, Paraná) (BARTSCH, 1920; TURNER, 1966; NAIR \& SARASWATHY, 1971; RANCUREL, 1971; BASTIDA \& TORTI, 1972; MULLER \& LANA, 1986).

Comentários: Espécie presente nas estações do ambiente estuarino nas capturas naturais (Estações I, II, III, IV, VII), estando ausente em ambiente marinho (Estação VI) e nas capturas em coletores de pinho (Estação V). Foi encontrada tanto em madeira submersa quanto em emersa, próxima às margens dos rios até distância aproximada de 250 a 300 
metros para o interior do bosque. Em troncos emersos esteve presente a uma altura máxima de $68 \mathrm{~cm}$. Ocorreu em todo o período pesquisado com valores salinos entre $0,16 \%$ a $20,3 \%$ e temperatura da água entre $22,5^{\circ} \mathrm{C}$ a $27,5^{\circ} \mathrm{C}$.

Psiloteredo healdi Bartsch, 1922

Figura 2. Psiloteredo healdi 1-4, faces externa e interna de dois pares de paleta.

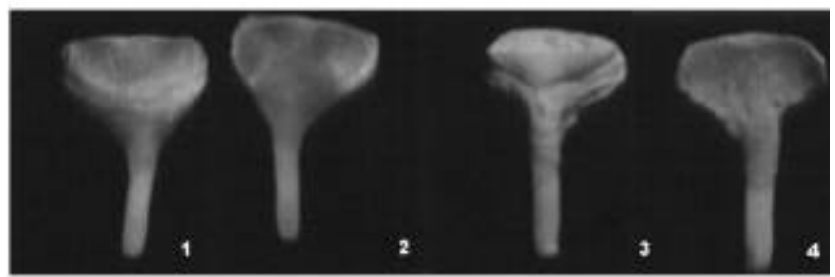

Sinônimos: Teredo (Neoteredo) healdi (Bartsch, 1931). Bartsch, 1931:2 (apud CLAPP

\& KENK, 1963:129; TURNER, 1966:104).

Localidade tipo: Cabimas, aproximadamente $0,5 \mathrm{Km}$ a sudeste de Maracaíbo, Venezuela. (apud CLAPP \& KENK, 1963:129; TURNER, 1966:104).

Material examinado: Foram examinados 47 espécimens coletados em quatro estações Distribuição Geográfica: Golfo do México, Antilhas, Panamá, Venezuela, Brasil (Alagoas, São Paulo).(BARTSCH, 1922; TURNER, 1966, 1971b; NAIR \& SARASWATHY,1971; LOPES, 1991).

Descrição: Partes moles branco-leitosa. Valvas com perióstraco de cor ferrugem recobrindo as valvas. Paletas sólidas, grossas, inteiramente calcárias, compactas, não segmentadas; lâminas com forma variável; face externa da lâmina com nítida depressão em forma de unha, com incrustações calcárias esbranquiçadas; face interna lisa, ligeiramente côncava, com típica coloração ferrugem quase uniforme, consis tência pesada; pedúnculo geralmente com discreta sinuosidade, com divisão na porção mais distal. Sifões unidos em toda extensão exceto na extremidade distal; tentáculos pequenos, pigmentados.

Comentários: Os exemplares de $P$. healdi foram coletados apenas nas margens do bosque, nas estações mais interiores do estuário (Estações I, II, III, VII) onde a salinidade variou entre $0,05 \%$ a $15,0 \%$ e a temperatura da água entre $22,5^{\circ} \mathrm{C}$ e $27,5^{\circ} \mathrm{C}$. 


\section{Teredo bartschi Clapp, 1923}

Figura 3. Teredo bartschi . 1-4, faces externa e interna de dois pares de paleta.

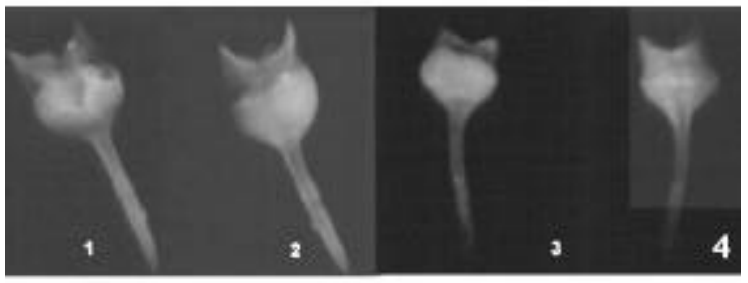

Sinônimos: Teredo fragilis Tate, 1888 (TATE, 1889:60-66, apud CLAPP \& KENK, 1963:960)

Teredo (Teredo) batilliformis Clapp, 1924 (CLAPP, 1924:282-284, apud CLAPP \& KENK, 1963: 226)

Teredo balatro Iredale, 1932 (IREDALE, 1932: 31, apud CLAPP \& KENK, 1963:494).

Teredo shawi Iredale, 1932 (IREDALE, 1932: 30, apud CLAPP \& KENK, 1963:494).

Teredo aegyptia Roch, 1935 (ROCH, 1935: 267-68 apud CLAPP \& KENK,1963:846)

Teredo (Teredo) grobbai Moll, 1937 (MOLL, 1937: 182 apud TURNER,1966).

Teredo (Teredo) hiloensis Edmondson, 1942 (EDMONDSON, 1942:113). Localidade tipo: Port Tampa, Flórida (apud CLAPP \& KENK, 1963: 225; TURNER, 1966: 90).

Material examinado: Foram examinados 207 espécimens coletados em coletores de pinho apenas na Estação V (desembocadura).

Distribuição Geográfica: Atlântico Ocidental: Carolina do Sul; Sul da Flórida; Texas: Golfo do México; Bermudas; Antilhas; Brasil ( Alagoas, Bahia, Rio de Janeiro, São Paulo, Paraná); Uruguai; Mediterrâneo: Mar Mediterrâneo; Indo-Pacífico: Madagáscar; Mar Vermelho; Golfo Pérsico; Índia ( Costa Oeste e Sul); Austrália, Nova Zelândia, Pacífico Central: Ilhas Mildway; Ilhas Havaí; Pacífico Oriental: Califórnia (introduzida) ( EDMONDSON, 1942; NAGABHUSHAMAN, 1955; NAIR \& SARASWATHY, 1971; MILANO \& LOPES, 1977-78; NAIR \& DHARMARAJ, 1980; CALVO, 1984).

Descrição:Paletas calcárias em forma de taça larga, sem segmentação. Lâmina com porção basal calcária branca e porção distal com capa de perióstraco transparente, amarelo claro e com prolongamentos laterais localizados distalmente. Espécimens jovens com divisão nítida entre a porção distal e basal da lâmina formando uma 
"ampulheta"; face externa suavemente côncava, lisa sem granulações; face interna plana; extremidade distal da lâmina em U largo; pedúnculo curto, geralmente sinuoso. Sifões separados, presença de papilas pigmentadas na face externa, sendo mais nítida na porção interna entre o sifão exalante e inalante.

Comentários: Teredo bartschi foi coletado em coletores de pinho submersos e emersos periodicamente. O nível salino esteve comprendido entre $25,74 \%$ e $33,82 \%$ e a temperatura da água compreendida entre $26,0^{\circ} \mathrm{C}$ e $29,0^{\circ} \mathrm{C}$.

Teredo clappi Bartsch, 1923

Figura 4. 1. Região Posterior (sifões); 2. Região Posterior (paletas); 3-5. Dois pares de paleta muito modificados.

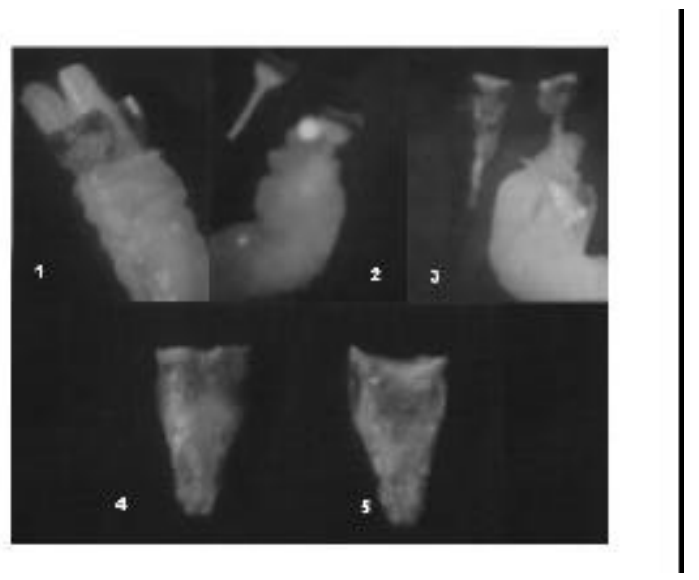

Sinônimos: Teredo trulliformis Miller, 1924 ( MILLER, 1924:150, apud CLAPP \& KENK, 1963:678; TURNER, 1966:126)

Teredo hermitensis Roch, 1929 (ROCH, 1929: 14; apud CLAPP \& KENK, 1963:843; TURNER, 1966:104).

Teredo adanensis Roch, 1935 (ROCH, 1935:265 apud CLAPP \& KENK, 1963:846; TURNER, 1966: 86).

Teredo renschi Roch, 1935 (ROCH, 1935:267 apud CLAPP \& KENK, 1963:846; TURNER, 1966:119).

(?) Teredo horsti "Roch"Moll, 1941 (MOLL, 1941:176 apud CLAPP \& KENK, 1963:698; TURNER, 1966:104).

Localidade tipo: Key West, Flórida (BARTSCH, 1923: 96-97 apud CLAPP \& KENK, 1963:128;TURNER, 1966:94).

Material examinado: Foram coletados 67 espécimens de Teredo clappi, apenas na Estação VI, ambiente marinho (mediolitoral) em troncos naturais.

Distribuição Geográfica: Atlântico Ocidental: Flórida; Antilhas; Brasil (Alagoas); IndoPacífico: Madagáscar; Mar Vermelho; Golfo Pérsico; Costa oeste, leste e sul da Índia; Indonésia; Austrália; Nova Guiné; Pacífico Central: Havaí (BARTSCH, 1923; EDMONDSON, 1942; NAGABHUSHANAN, 1955; NAIR, 1965; 
SANTHAKUMARAN, 1966; 1976; NAIR \& SARASWATHY, 1971; TURNER \& JOHNSON,1971; HADERLIE, 1974; SANTHAKUMARAN \& PILLAI, 1974; DHARMARAJ \& NAIR, 1980; KRISHNAN, JAIN \& TEWARI, 1980; SANTHAKUMARAN \& JAIN, 1981; NAIR \& DHARMARAJ, 1983; ABBOTT, 1984).

Descrição: Paletas em forma de taça, totalmente calcárias, de aspecto consistente. Lâmina envolvida na porção mais distal por um anel de coloração ferrugem; face externa com bifurcação na extremidade distal, em forma de $U$ aberto; pedúnculo com suave curvatura, comprimento variável, com divsão próxima à base da lâmina. Sifões exalante e inalante com faixas horizontais, extremidades sem pigmentação. Comprimento do maior exemplar inteiro examinado foi de $45,0 \mathrm{~mm}$ e o menor $9,0 \mathrm{~mm}$.

Comentários: Esta espécie foi coletada apenas em ambiente marinho (mediolitoral) perfurando troncos em decomposição de Cocus nucifera (coqueiro-da-baía) e em Machaerium sp (coração-de-negro). A salinidade esteve compreendida entre 30,95\% a $35,26 \%$ e a temperatura da água entre $25,5^{\circ} \mathrm{C}$ a $27,0^{\circ} \mathrm{C}$.

Lyrodus floridanus (Bartsch, 1922)

Figura 5. Lyrodus floridanus. 1. Espécime inteiro.

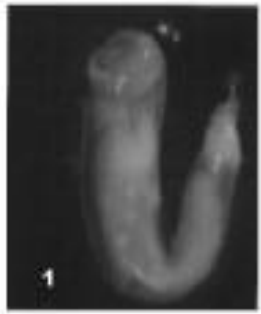

Sinônimos: Teredo (Teredops) floridana Bartsch, 1922 (BARTSCH, 1922: 28)

Teredo pedicellatus (Quatrefages, 1849) (QUATREFAGES, 1849: 26-27 apud CLAPP \& KENK, 1963:804).

Localidade tipo: Tampa, Flórida (BARTSCH, 1922:29).

Material examinado: Foram examinados onze espécimens.

Distribuição Geográfica: Atlântico Ocidental: Flórida; Brasil (Alagoas, Rio de Janeiro, São Paulo, Paraná) (BARTSCH, 1922; TURNER, 1966; MULLER, 1984; MULLER \& LANA, 1986; 1987; JUNQUEIRA, SILVA \& SILVA, 1989; JUNQUEIRA \& SILVA, 1990;LOPES, 1991).

Descrição: Paletas não segmentadas, forma de remo estreito na porção basal; extremidade distal em forma de $\mathrm{V}$ arredondado e profundo, com dois prolongamentos (expanções do perióstraco); face interna com "mancha" arredondada típica ("ampulheta" por MULLER, 1984); face externa com faixas (linhas horizontais) muito finas; perióstraco marrom-castanho escuro, brilhante; pedúnculo curto a medianamente longo com ligeira inclinação; sifões separados, com papilas laranja-escuro na extremidade. Maior exemplar com 20,0 mm e menor com 8,0 mm. 
Comentários: Os exemplares desta espécie foram coletados apenas na área de desembocadura (Estação V) em coletores de pinho sempre submersos. O registro de salinidade esteve entre $25,74 \%$ e $33,82 \%$; temperatura da água esteve entre $26,0^{\circ} \mathrm{C}$ e $27,0^{0} \mathrm{C}$.

Subfamília Bankiinae Turner, 1866.

\section{Nototeredo sp}

Figura 6. Nototeredo sp. 1-6, faces externa e interna de três pares de paleta.

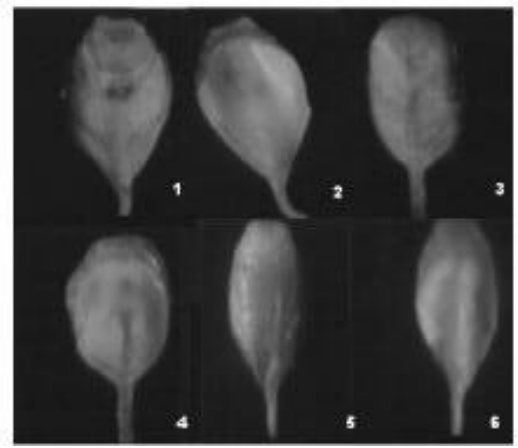

Gênero Nototeredo Bartsch, 1923: 100-101 apud CLAPP \& KENK, 1963: 128.

Espécie Tipo: Teredo (Nototeredo) edax Hedley, 1825: 501 apud CLAPP \& KENK, 1963: 128.

São conhecidas três espécies para o gênero: Nototeredo norvagica (Spengler,1792: 102103); $\quad$ Nototeredo edax (Hedley, 1895: 501-505); Nototeredo knoxi (Bartsch, 1917: 48-50); apud CLAPP \& KENK, 1963 : 929; 447; 125.

Material examinado: Foram examinados 22 espécimens.

Descrição: Paletas com lâminas segmentadas (segmentos fusionados), forma variada de estreita a larga, perióstraco amarelo-claro; face externa ligeiramente côncava, porção distal com faixas concêntricas e material calcário "friável'; face interna côncava e lisa; pedúnculo translúcido, pequeno, retorcido tênue estendendo-se no meio da face interna da lâmina. Sifões separados com linhas horizontais paralelas na porção basal; sifão exalante e inalante com muitos tentáculos pequenos.

Comentários; Nos exemplares examinados não foram observados nenhum dos três tipos de tentáculos (diagnósticos para as três espécies de Nototeredo) que se estendem do sifão exalante.

Os exemplares foram coletados apenas no ambiente marinho (mediolitoral0 em troncos de Eschweilera sp (currais), Machaerium sp (madeira flutuante) e em troncos de Cocus nucifera.Os registros para salinidade estiveram entre $30,95^{\circ}{ }^{\circ o}$ e $35,26^{\circ} \%^{\circ o}$ : temperatura da água esteve entre $25,5^{\circ} \mathrm{C}$ e $27,0^{\circ} \mathrm{C}$. 
Figura 7. Nausitora fusticula. 1-6 faces externa e interna de quatro pares de paleta; 7. sifões.

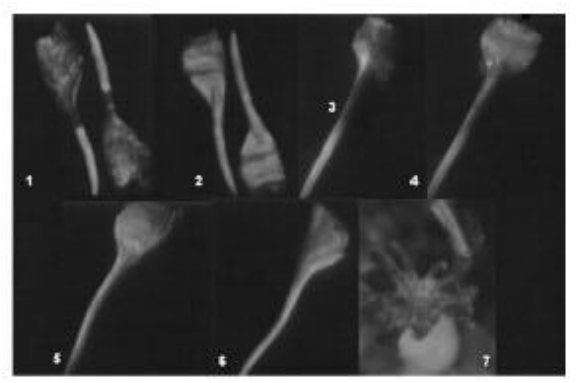

Sinônimos: Teredo fusticulus Jeffreys, 1860 (JEFFREYS, 1860:125 apud CLAPP \& KENK, 1963:504; TURNER, 1966:102).

Bankia (Naussitora) brasiliensis Bartsch, 1922 (BARTSCH, 1922:15)

Bankia (Nausitora) excolpa Bartsch, 1922 (BARTSCH, 1922:13)

Localidade tipo: Em troncos flutuantes de Cedrella odorata Griseb. (cedro), em Leith, Escócia, provavelmente provenientes da região das Antilhas (apud CLAPP \& KENK, 1963 ; TURNER, 1966:102).

Material examinado: Foram examinados 611 exemplares coletados em cinco estações do estuário-manguezal.

Descrição: Coloração castanha (clara a escura) com aspecto brilhante. Paletas com lâminas segmentadas em cones parcialmente fusionados; face externa com capa periostracal amarelo-laranja de cor ferrugem e com papilas na porção distal; face interna dividida em uma porção distal, com segmentos nítidos e porção basal lisa; pedúnculo alongado, reto, algumas vezes com discreta sinuosidade. Sifão inalante com doze longos tentáculos de margens recortadas.

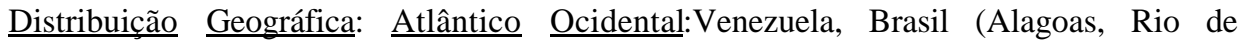
Janeiro, São

Paulo, Paraná); Uruguai. Pacífico Oriental: Costa Leste do Panamá; Peru. Indo-Pacífico: Costa Leste da Índia (Estuário de Mahanadi) (BARTSCH, 1922; TURNER, 1966; 1971a; SUBBARAO, 1968; NAIR \& SARASWATHY, 1971; RIOS, 1975; BASTIDA \& TORTI, 1972; NAIR, 1975; MULLER, 1984; RIOS, 1985; MULLER \& LANA, 1986; 1987; LOPES, 1991; CORREIA, 1992).

Comentários: A espécie foi coletada em troncos naturais em todas as estações do manguezal (Estações I, II, III, IV, VII) estando ausente no ambiente marinho (Estação V) e também nos coletores de pinho (Estação VI). A salinidade esteve situada entre $0,04 \%$ a $20,3 \%$ e a temperatura da água apresentou-se entre $22,5^{\circ} \mathrm{C}$ a $27,5^{\circ} \mathrm{C}$. 
Bankia fimbriatula Moll e Roch, 1931

Figura 8. Bankia fimbriatula. 1-2 faces externa e interna de um par de paleta; 3-4 cones

(paleta)

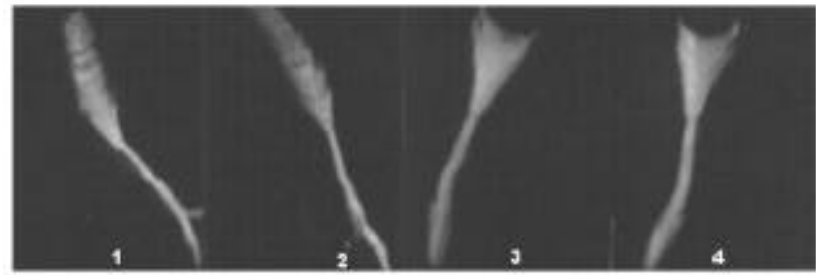

Sinônimos: Teredo fimbriata Jeffreys, 1860 (JEFFREY, 1860:126 apud CLAPP \& KENK, 1963:504; TURNER, 1966:101).

Bankia (Bankia) canalis Bartsch, 1944 (BARTSCH, 1944:1 apud CLAPP \& KENK, 1963:93; TURNER, 1966:93).

Localidade tipo: Britsch Isles (apud CLAPP \& KENK, 1963:213-214).

Material examinado: Foram examinados 95 espécimens (Tabela ?).

Distribuição Geográfica: Atlântico Ocidental: Flórida (Costa Leste/Oeste), Golfo do México; Antilhas,; Honduras; Nicarágua; Costa Rica; Panamá; Venezuela; Brasil (Pernambuco, Alagoas, Bahia, Rio de Janeiro, São Paulo, Paraná). Atlântico Oriental: Costa Atlântica da Europa. Pacífico Oriental: Costa Oeste do Panamá. Indo-Pacífico: Costa da Índia (Estuário de Mahanadí) (SUBBARAO, 1968; CLENCH \& TURNER, 1946; NAIR \& SARASWATHY, 1971; TURNER \& JOHNSON, 1971; NAIR, 1975; MILANO \& LOPES, 1977-78; ABBOTT, 1984; MULLER, 1984; MULLER \& LANA, 1986; CORREIA, 1992; CLENCH \& TURNER, 1946; RIOS, 1970; 1975; 1985; SILVA, 1985; MARTINS-SILVA, SILVA \& JUNQUEIRA, 1985; JUNQUEIRA, SILVA \& MARTINS-SILVA, 1985; JUNQUEIRA, 1986;SILVA, SILVA \& JUNQUEIRA, 1988; JUNQUEIRA, SILVA \& SILVA, 1989; SILVA, JUNQUEIRA \& SILVA, 1990; LOPEZ, 1985; TIAGO, 1989; LOPES, 1991; MULLER, 1984; MULER \& LANA, 1986.

Descricão: Paletas alongadas e segmentadas em cones não fusionados; lâmina cônica com porção calcária em V, com imbricação sem espaços; perióstraco amarelo-laranja ou transparente com bordos serrilhados, longos, nos cones da face interna e curtos, na face externa; prolongamentos bifurcados do perióstraco sempre visível em ambas as extremidades de cada cone; pedúnculo longo, estendendo-se por toda lâmina. Sifões longos, separados com papilas de castanho a roxo nas extremidades.

Comentários: Bankia fimbriatula foi coletada em ambiente estuarino em troncos de Laguncularia racemosa (mangue manso) e em coletores de pinho e no ambiente 
marinho em troncos de coqueiro. A salinidade esteve compreendida entre $22,5 \%$ a $35,26 \%$ e a temperatura da água entre $25,5^{\circ} \mathrm{C}$ a $27,0^{\circ} \mathrm{C}$.

\section{DISCUSSÃO}

Na identificação das espécies de Teredinidae levou-se em consideração para a definição genérica e específica a morfologia das paletas, estruturas características dessa família, localizadas na região posterior.

Nas espécies pesquisadas, as paletas apresentaram-se com variações significativas entre os indivíduos de uma mesma espécie. MILLER (1926), TURNER (1966) e RANCUREL (1971) se referem a variabilidade dessas estruturas à condicionamentos genéticos, idade e influências fisiológicas, mecânicas e ambientais.

Em Teredo clappi foram observadas variabilidade intraespecífica marcante entre as paletas da maioria dos 67 espécimes coletados; variações moderadas em Nausitora fusticula e Neoteredo reynei, e diferenças pouco notáveis entre as paletas de Teredo bartschi, Lyrodus floridanus, Psiloteredo healdi e Bankia fimbriatula.

As variações nas paletas de Teredo clappi foram principalmente relacionadas por desgastes acentuados e acúmulo de partículas aglutinadas. Dentre os 67 espécimes examinados ocorreram paletas desde a forma íntegra, comum a espécie até totalmente diferente da forma original, tendo sido também constatadas variações marcantes em um mesmo par de paletas. Neoteredo reynei e Nausitora fusticula apresentaram diferenciações intraespecíficas através de desgastes e diferenças na coloração. Teredo bartschi e Lyrodus floridanus apresentaram variações principalmente entre indivíduos jovens e idosos. Psiloteredo healdi e Bankia fimbriatula foram as espécies que demonstraram maior uniformidade em relação à forma das paletas.

\section{CONCLUSÕES}

A família Teredinidae esteve representada no Estuário do Rio Manguaba e ambiente marinho (mediolitoral) da Praia de Barreiras do Boqueirão (litoral norte de Alagoas) por oito espécies distribuídas em sete gêneros o que indica uma boa representação, considerando que para a costa brasileira há referência de vinte e cinco espécies distribuídas em oito gêneros.

Neoteredo reynei, Psiloteredo healdi, Teredo bartschi, Lyrodus floridanus e Nototeredo sp. constam como primeira ocorrência para o litoral de Alagoas, enquanto Teredo clappi como primeiro registro para o litoral brasileiro.

$\mathrm{Na}$ área estuarina, Nausitora fusticula foi a espécie de maior freqüência relativa, seguida de Neoteredo reynei e Teredo bartschi, enquanto Psiloteredo healdi e Bankia fimbriatula apresentaram menor freqüência.

No ambiente marinho, T. clappi apresentou a maior freqüência predominando em relação à $B$. fimbriatula e Nototeredo sp.

\section{REFERÊNCIAS BIBLIOGRÁFICAS}

ABBOTT,R.J.1974. American Seashells. 2 ed. New York: Van Nostrand Reinhold $663 p$.

BARTSCH, P. 1920. A new shipworm. Proc. Biol. Soc. Wash. v. 33, p. 69-70. 
BARTSCH, P. 1922. A monograph of the American Shipworms. Bull. U. S. Nat. Mus. Washington, v. 122, p. 1-51.

BASTIDA, R. \& TORTI, M. R. 1972. Organismos perforantes de las costas argentinas. I. La presencia de Lyrodus pedicellatus (Quatrefages, 1849) (Mollusca, Pelecypoda) en el Puerto de Mar del Plata. Clave para el reconocimiento de los Teredinidae sudamericanos. Physis, Buenos Aires, v. 31, n.82, p. 39-50.

CALVO, G. 1984. Ataques de organismos perforantes a 6 espécies de maderas expuestas al medio marino. Contrib. Depto. Oceanogr. (F.H.C.). Montevideo, v. 1, n. 3, p. 1-7.

CLAPP, W. F. \& KENK, R. 1963. Marine Borers an Annotated Bibliogaphy. Office of Naval Research. Department of the Navy. Washington, 113p.

CLENCH, W. \& TURNER, R.D. 1946. The Genus Bankia in the western Atlantic. Johnsonia, Cambridge, v.2, n. 19, p. 1-28.

CORREIA, M. D. 1992. Fauna associada aos troncos de madeira do Complexo Estuarino-lagunar Manguaba. IN: Congresso Brasileiro de Zoologia, 15 / Congresso Latino-Americano de Zoologia, 12, Belém, julho/1992. Resumos ... Belém, p. 177.

DHARMARAJ, J. \& NAIR, N.B. 1980. Wood-boring organisms in relation to aquaculture along the coasts of India. IN. SANTHAKUMARAN, L. N. (Org.). Marine Woord borers of India an annotated bibliography. Goa, India: Forest Research Laboratory, 1985. 146 p. p. 26-27.

EDMONDSON, C.H. 1942. Teredinidae of Hawaii. Occasional Papers of Bernice P. Bishop Museum. Honolulu. v. 17. n. 10, p. 97-148.

HARDELIE, E.C. 1974. Wood boring marine animals from the Gulf of Elat. Isr. J. Zool. , v. 23, p. 57-59.

HOAGLAND, K. E. \& TURNER, R.D. 1981. Evolution and adaptive Radiation of Wood-boring Bivalves (Pholadacea). Malacologia. v.21, n.1-2, p.111-148.

JUNQUEIRA, A. de O.R. 1986. Estudo Experimental dos Teredinidae Rafinresque, 1815 (Mollusca: Bivalvia do Estuário da Lagoa da Tijuca, Rio de Janeiro, RJ, Brasil. Rio de Janeiro. Universidade Federal do Rio de Janeiro. 252p. Dissertação (Mestrado em Ciências Biológicas-Zoologia-UFRJ).

JUNQUEIRA, A. de O.R., SILVA, S.H.G. \& MARTINS-SILVA, M.J. 1985. Teredinidae (Mollusca: Bivalvia) no litoral do Rio de Janeiro. II. Lagoa da TijucaBarra da Tijuca. IN: Congresso Brasileiro de Zoologia, 12, Campinas, janeirofevereiro/ 1985. Resumos ... Campinas, p. 29.

JUNQUEIRA, A. de O.R., SILVA, S.H.G. \& MARTINS-SILVA, M.J. 1989. Avaliação da infestação e diversidade de Teredinidae (Mollusca: Bivalvia) ao longo da costa do Estado do Rio de Janeiro, Brasil. Mem. Inst. Oswaldo Cruz. Rio de Janeiro, v. 84, Supl. 4, p. 275-280.

KRISHNAN, R.V., JAIN, J.C. \& TEWARI, M. C. 1980. A review of recent investigation on the protection of timber in sea waters in India. IN: SANTHAKUMARAN, L. N. (Org.). Marine Wood-borers of India an annotated bibliography. Goa, India: Forest Research Laboratory, 1985. 146p. p. 48-49.

LOPES, S.G.B. C. 1991. Sobre a Anatomia Funcional de Nausitora fusticulata (Jeffreys, 1860(Mollusca-Bivalvia) e Contribuição ao Conhecimento dos 
Teredinidae do Manguezal da Praia Dura, Ubatuba (SP), Brasil. São Paulo. Universidade de São Paulo. 130p. Tese (Doutorado em Ciências-Zoologia-Instituto de Biociências - USP).

LOPEZ, G.A.C.1985. Development of marine borers research in São Paulo, Brazil. 8p. (Trabalho apresentado na $16^{\text {th }}$ Anual Meeting of the Marine Wood Preservation, 1985, Guarujá, SP.)

MARTINS-SILVA, M.J. SILVA, S.H.G. \& JUNQUEIRA, A. de O.R. 1985. Teredinidae (Mollusca: Bivalvia) no litoral doEstado do Rio de Janeiro. III. Baía de Sepetiba. IN: Congresso Brasileiro de Zoologia, 12, Campinas, janeiro-fevereiro /1985. Resumos ... Campinas, p.30.

MILANO, S. \& LOPEZ, G.A.C. 1977-78. Resistência Natural de Madeiras e Xilófagos Marinhos: resutados da primeira avaliação após três meses de instalação do ensaio.

Preservação de Madeiras. São Paulo, v. 8/9, n. 1, p.31-42.

MUlleR, A. C. de P. 1984. Organismos Marinhos Perfuradores de Madeira do

Estado do Paraná. Curitiba. Universidade Federal do Paraná. 111p. Dissertação (Mestrado em Zoologia - Setorde Ciências Biológicas - UFPR).

MULlER, A. C. de P \& LANA, P. da C. 1986. Teredinidae (Mollusca: Bivalvia) do litoral do Paraná, Brasil. Nerítica. Pontal do Sul., v.1, n.3, p. 27-48.

MULlER, A. C. de P \& LANA, P. da C. 1987. Padrões de Distribuição Ge ográfica de Teredinidae (Mollusca: Bivalvia) do Estado do Paraná. Ciência e Cultura. São Paulo., v. 39, n. 12, p. 1175-1177.

NAGABHUSHANAM, R. 1955. A systematic account of the Molluscan wood-borers of Visakhapatnam Harbour. IN: SANTHAKUMARAN, L. N. (Org.). Marine wood-borers of India an annotated bibliography. Goa, India: Forest Research Laboratory, 1985. 146p. p. 58.

NAIR, N.B. 1965. Marine timber-boring organismsof the Indian Coast. Report on a collection from the southeast coast of India with notes on the distribution in the Indo-Pacific area. IN: SANTHAKUMARAN, L. N. (Org.). Marine wood-borers of India an annotated bibliography. Goa, India: Forest Research Laboratory, 1985. 146p. p. 73-74..

NAIR, N.B. 1975. Shipworms of Venezuela, report on a collection form the Gulf of Cariago. Bol. Inst. Oceanogr. Univ. Oriente. Cumaná, Venezuela, v. 14, n.1, p.129-146.

NAIR, N.B \& DHARMARAJ, K. 1980. Wood-boring molluscs of the Palk Bay and the Gulf of Mannar. IN: SANTHAKUMARAN, L. N. (Org.). Marine wood-borers of India an annotated bibliography. Goa, India: Forest Research Laboratory, 1985. 146p. p. 78.

NAIR, N.B \& SARASWATHY, M. 1971. The biology of wood-boring teredinid molluscs. IN: Adv. Mar. Biol. RUSSEL, F.S. \& Yonge, M. (Eds.), v. 9, p. 335-509.

RANCUREL, P. 1971. Les Teredinidae (Mollusques :Lamellibranches) dans les lagunes de Côte d'Ivoire. Mémoires Orstom, v. 47, Paris, 212p.

RIOS, E. C. 1970. Coastal Brazilian Seashells. Fundação Cidade do Rio Grande, Museu Oceanográfico do Rio Grande. Rio Grande, 255p. 
RIOS, E. C. 1975. Brazilian Marine Mollusks Iconography. Fundação Universidade do Rio Grande, Centro de Ciências do Mar, Museu Oceanográfico. Rio Grande. $331 \mathrm{p}$.

RIOS, E. C. 1985. Seashells of Brazil. Fundação Cidade do Rio Grande. Fundação Universidade do Rio Grande. Museu Oceanográfico. Rio Grande, 328 p.

SANTHAKUMARAN, L. N. \& JAIN, J. C. 1981. Observations on the destruction of fishing craft in India by marine wood-borers whit special reference to the west coast. IN: SANTHAKUMARAN, L. N. (Org.). Marine wood-borers of India an annotated bibliography. Goa, India: Forest Research Laboratory, 1985. 146p. p.110.

SANTHAKUMARAN, L. N. \& PILLAI, S. R. M. 1974. Incidence of marine woodborers in mangroves in the vicinity of Bombay harbour. IN: SANTHAKUMARAN, L. N. (Org.). Marine wood-borers of India an annotated bibliography. Goa, India: Forest Research Laboratory, 1985. 146p. p.111.

SILVA, S.H.G. da. 1985. Estudo Experimental sobre a Infestação de Perfurantes Marinhos de Madeira da Costa do Estado do Rio de Janeiro. São Paulo. Universidade de São Paulo. 231p. Tese (Doutorado em Ciências-Fisiologia AnimalInstituição de Biociências - USP).

SILVA, M.J., SILVA, S.H.G. \& JUNQUEIRA, A. de O.R.1988. Distribuição Vertical de Teredinidae (Mollusca, Bivalvia) em Portogalo, Angra dos Reis, Rio de Janeiro, Brasil. Rev. Bras. de Zool., v. 5, p. 155-165.

SUBBARRAO, N.V. 1968. Report on a collection of woood-boring Molluscs from Mahanadi estuary, Orissa, India. IN: SANTHAKUMARAN, L. N. (Org.). Marine wood-borers of India an annotated bibliography. Goa, India: Forest Research Laboratory, 1985. 146p. p.135.

TIAGO, E.G. 1989. Sobre uma comunidade de Teredinidae (Mollusca, Bivalvia) em São Sebastião (SP), Brasil. São Paulo, Universidade de São Paulo. 88p. Dissertação (Mestrado em Ecologia- Instituto de Biociências - USP).

TURNER, R.D. 1966. A Survey and Illustrated Catalogue of the Teredinidae (Mollusca: Bivalvia). Cambridge: The Museum of Comparative Zoology, 265p.

TURNER, R.D. 1971a. Identification of Marine Wood-Boring Molluscs. IN: Marine Borers, Fungi and Fouling Organisms of Wood. Paris, E. B. G. Jones and S. K. Eltringham, Cap. 1, p.17-64.

TURNER, R.D. \& JOHNSON, A. C. 1971. Biology of Marine Wood-Boring Molluscs. IN: Borers, Fungi and Fouling Organisms of Wood. Paris, E. B. G. Jones and S. K. Eltringham, Cap. 13, p. 259-301. 